ARTIGO

ORIGINAL

\title{
Estabilidade no prognóstico do perfil de aptidão física de jovens atletas de handebol
}

\author{
Vagner Raso ${ }^{1}$, Carlos Frederico d'Avila Brito ${ }^{1}$, Timóteo Leandro Araújo ${ }^{1}$, \\ Vagner Roberto Bergamo ${ }^{2}$, Douglas Roque Andrade ${ }^{1}$, Sandra Marcela Mahecha Matsudoㄹ, \\ Nanci Maria de França ${ }^{3}$ e Victor Keihan Rodrigues Matsudo ${ }^{1}$
}

\section{RESUMO}

Este estudo teve como objetivo: 1) verificar a estabilidade do perfil de aptidão física de jovens atletas de handebol durante a adolescência; e 2) comparar os resultados com a seleção paulista adulta feminina. Para tanto, a amostra foi constituída por cinco jovens atletas de handebol feminino que foram seguidas semilongitudinalmente por dois anos, com média de idade e tempo de prática inicial e final de $14,2 \pm 1,3$ anos, $18,4 \pm 10,7$ meses e $16,2 \pm 1,3$ anos, 42,8 $\pm 10,7$ meses, respectivamente $(12 \mathrm{~h} / \mathrm{semana}$ de treinamento). Na temporada de 1997 quatro atletas foram pré-selecionadas para a seleção brasileira de cadetes. As variáveis mensuradas foram: peso corporal (P); altura total (A); impulsão vertical sem (IVS) e com auxílio dos membros superiores (IVC); impulsão horizontal (IH); agilidade (SR); velocidade $(50 \mathrm{~m})$ e potência anaeróbica (40seg.), seguindo padronização CELAFISCS. Os coeficientes de Pearson e Spearman foram significativamente altos e muitos altos $(\mathrm{p}$ $<0,05)$ para $\mathrm{P}(0,88)$, A $(0,98$ e 0,99$), 50 \mathrm{~m}(0,95$ e 0,90$)$ e 40seg. $(0,87$ e 0,90$)$. Estes dados permitem concluir que as jovens atletas apresentaram alta estabilidade nas variáveis antropométricas, velocidade e potência anaeróbica durante um período de dois anos da adolescência, favorecendo a hipótese de que a expectativa de sucesso esportivo pode ser predita em idades precoces.

Palavras-chave: Detecção de talentos. Talento esportivo. Estratégia Z-CELAFISCS. Esporte. Estabilidade. Adolescência. Handebol.

1. Centro de Estudos do Laboratório de Aptidão Física de São Caetano do Sul - CELAFISCS.

2. Universidade Metodista de Piracicaba - UNIMEP e Pontifícia Universidade Católica de Campinas - PUCCAMP.

3. Universidade Católica de Brasília - UCB.

Endereço para correspondência:

Vagner Raso

Rua Marquês de Praia Grande, 540, apto. 32B

03129-110 - São Paulo, SP

E-mail: vraso@usa.net

Rev Bras Med Esporte - Vol. 6, № 3 - Mai/Jun, 2000

\section{ABSTRACT \\ Stability in the physical fitness profile prognosis of young handball athletes}

The purpose of this study was 1) to verify the stability of the physical fitness profile of young handball athletes during adolescence, and 2) to compare these athletes to the São Paulo state handball adult team. The sample comprised five young female handball athletes who were followed-up for two years, with mean age and practice time before and after of $14.2 \pm 1.3$ years-old, $18.4 \pm 10.7$ months and 16.2 \pm 1.3 years-old, $42.8 \pm 10.7$ months, respectively $(12 \mathrm{~h} /$ week training). Variables measured were: body weight $(W)$; body height $(H)$; vertical jump without (VJWo) and with help of arms (VJ); long jump (LJ); agility (SR); velocity (50m) and anaerobic power (40seg.), by CELAFISCS standardization. It was verified that with the exception of VJW and $50 \mathrm{~m}$, the young athletes showed upper values for all variables. The Pearson and Spearman coefficients were significantly higher and much higher $(p<0.05)$ for $W(0.88), H(0.98$ and 0.99), $50 \mathrm{~m}$ (0.95 and 0.90) and $40 \mathrm{sec}(0.87$ and 0.90 ). Data permit to conclude that young athletes showed stability in anthropometric variables, velocity, and anaerobic power during the adolescence period, thus supporting the hypothesis that the expectation of success in sports can be predicted from early age.

Key words: Talent detection. Athletic talent. Z-CELAFISCS strategy. Sports. Stability. Adolescence. Handball.

\section{INTRODUÇÃO}

No campo da ciência e do movimento o desafio em predizer o futuro tem recebido a atenção de muitos pesquisadores $^{1-4}$. Hollmann e Hettinger ${ }^{5}$ citam que, de 1.000 garotos na faixa etária de 10 anos, apenas um irá apresentar valores de $\dot{\mathrm{V}}{ }_{2 \max }$ de $70 \mathrm{ml} . \mathrm{kg}^{-1} \cdot \mathrm{min}^{-1}$, sendo que o valor padrão referencial nacional para esta idade é $46,33 \mathrm{ml} . \mathrm{kg}^{-1}$. $\min ^{-16}$.

A hereditariedade pode afetar a performance de um indivíduo durante o período de detecção e de acompanha- 
mento. Wilson e Poster ${ }^{7}$ verificaram que pares de gêmeos monozigóticos (MZ) seguidos do primeiro ao nono ano de vida apresentam valores similares para a altura $(r=0,86$ a $0,95)$ e peso corporal $(r=0,35$ a 0,89$)$. Os valores de correlação da idade de menarca de pares de gêmeos MZ são maiores $(0,80)$ que a de pares de gêmeos dizigóticos $(0,60)^{8}$.

De acordo com Malinowski ${ }^{9}$, a variabilidade genética é responsável pelo desenvolvimento dos indivíduos excepcionais, como, por exemplo, aqueles que apresentam características que os fazem muito diferentes da maioria da população, além do resultado de um programa de treinamento (fenótipo) que não é dependente apenas do genótipo e do ambiente, mas também está relacionado com a sensibilidade com que o genótipo responde ao ambiente (treinamento $)^{4}$.

Esta característica de heterogeneidade na treinabilidade está relacionada com o genótipo e é referida como interação treinamento-genótipo ${ }^{10}$. Simoneau et al. ${ }^{11}$ observaram que aproximadamente $70 \%$ da potência anaeróbica láctica é dependente da hereditariedade, enquanto a magnitude de resposta da potência aeróbica máxima ao treinamento de endurance é de $77 \%{ }^{12}$. Thomis et al. ${ }^{10}$ verificaram que o ganho de força muscular obtido através do teste de uma repetição máxima (1-RM) após 10 semanas de um programa de treinamento de resistência para os flexores do cotovelo em pares de gêmeos dizigóticos foi metade daquele observado em gêmeos MZ, indicando uma contribuição moderada dos fatores genéticos.

Mas durante o processo de detecção de talentos, a seleção do indivíduo que apresenta os melhores resultados em um determinado momento não garante necessariamente a manutenção deste mesmo posto durante todo o período da vida quando comparado com indivíduos de faixas etárias correspondentes. Por isso, vários estudos têm sido elaborados nos últimos anos para saber se a boa performance de uma criança aos 10-12 anos de idade pode ser mantida na vida adulta ${ }^{13-15}$.

Brito et al. ${ }^{16}$ acompanharam longitudinalmente um grupo de meninos dos 9,14 $\pm 1,86$ aos 14,0 $\pm 1,88$ anos de idade e analisaram as características das variáveis antropométricas durante esse período. Puderam observar que a maioria dos valores obtidos na adolescência de peso corporal, altura, circunferências de braço e perna, estava sendo explicada pelos resultados observados na infância. Araújo et al. ${ }^{17}$ verificaram altos e significativos valores de estabilidade na transição da infância para a adolescência em um grupo de 61 garotas. Em outro estudo, também foi observado que crianças sob risco nutricional localizadas na média da população da mesma característica apresentam melhores resultados de estabilidade para as variáveis an- tropométricas, agilidade e força de membros superiores que crianças localizadas nas extremidades da curva ${ }^{18}$.

Estes estudos procuram determinar o tracking ou stability, que pode ser definido como a capacidade que um indivíduo possui para manter a mesma posição perante o grupo durante determinado período de tempo ${ }^{3,19}$. Eles se caracterizam pela monitorização de uma determinada variável durante um período da vida, como, por exemplo, na transição da infância para a adolescência. Deste modo, tão importante quanto selecionar é determinar a possibilidade de estabilidade dos resultados observados. Portanto, este estudo teve como objetivos: 1) verificar a estabilidade de variáveis de aptidão física e indiretamente a expectativa de sucesso esportivo de jovens atletas de handebol durante o período da adolescência; e 2) comparar os resultados com a seleção paulista adulta feminina.

\section{MATERIAL E MÉTODOS}

\section{Amostra}

A amostra foi constituída por cinco jovens atletas de handebol feminino que foram seguidas semilongitudinalmente por dois anos. Um informe de consentimento que explicava o desenvolvimento experimental do estudo e permitia a utilização dos dados coletados foi obtido de cada atleta e do seu respectivo representante legal.

No início do estudo (setembro/1995) as médias de idade e de tempo de prática esportiva foram de 14,2 $\pm 1,3$ anos e de 18,4 $\pm 10,7$ meses, respectivamente. No final (setembro/1997), foram de 16,2 $\pm 1,3$ anos e de 42,8 $\pm 10,7$ meses, respectivamente. As atletas treinavam cerca de $12 \mathrm{~h}$ por semana. Todas as atletas no início do estudo já haviam tido o primeiro fluxo menstrual, sendo a idade média de menarca de $12,5 \pm 0,4$ anos.

Durante o período do estudo as atletas participaram dos Jogos Regionais da região de Campinas, em que se sagraram campeãs no ano de 1997 e ficaram em sétimo lugar nos Jogos Abertos do Interior do Estado de São Paulo ocorridos em Bragança Paulista no mesmo ano. Também disputaram o campeonato paulista da modalidade na categoria júnior e posicionaram-se em quarto lugar. Ressalta-se que quatro das atletas participantes do presente estudo foram pré-selecionadas para a seleção brasileira de cadetes na temporada de 1997.

\section{Medidas}

As variáveis mensuradas no início e no final do estudo foram: altura total, peso corporal e força de membros inferiores: através do teste de impulsão vertical sem (IVS) e com auxílio dos membros superiores (IVC) e impulsão horizontal (IH); agilidade: através do teste de shuttle-run (SR); 
velocidade: através do teste de 50 metros $(50 \mathrm{~m})$; e potência anaeróbica: através do teste de 40 segundos (40 seg.). Todos os testes seguiram a padronização CELAFISCS.

\section{Análise estatística}

Para interpretar os resultados foi utilizada a estatística descritiva através da medida de tendência central (média aritmética) e de dispersão (desvio padrão). $O$ índice $z$ foi determinado para localizar o posicionamento das jovens atletas assim como das atletas da seleção paulista de handebol feminino na curva de Gauss, de acordo com critérios padrões de referência ${ }^{20}$.

Os valores de $\mathrm{z}$ foram calculados conforme a fórmula que segue:

$$
\mathrm{z}=\frac{\mathrm{X}-\mathrm{u}}{\sigma}
$$

onde, $\mathrm{z}=$ distância da média da população ou critério padrão de referência, em unidades de desvio padrão; $\mathrm{X}=$ resultado do sujeito; $\mathrm{u}=$ média da população ou critério padrão de referência para o indivíduo da mesma idade e sexo; $\sigma=$ desvio padrão da população ou do critério padrão de referência para o indivíduo da mesma idade e sexo.

A análise dos dados da primeira (set/1995) com a segunda avaliação (set/1997) foi feita com o teste $t$ de Student para amostras dependentes, além do teste não paramétrico de Wilcoxon. E a comparação dos resultados das jovens atletas no $1^{\circ}$ e $2^{\circ}$ momento com os dados das atletas da seleção paulista (jovens atletas set/95 x seleção paulista e jovens atletas set/97 x seleção paulista) foi determinada através do teste $t$ de Student para amostras independentes.

A determinação do nível de associação entre as avaliações (set/1995 x set/1997) foi feita através do coeficiente de correlação linear de Pearson para medidas gausianas (r) acompanhada do coeficiente de explicação (R), para determinar quanto os resultados obtidos no momento final (set/ 1997) estariam sendo explicados pelos valores observados

TABELA 1

Valores absolutos e índice $z$ das variáveis antropométricas

\begin{tabular}{|c|c|c|c|c|c|c|}
\hline \multirow[b]{3}{*}{ Variáveis } & \multicolumn{6}{|c|}{ Jovens atletas } \\
\hline & \multicolumn{2}{|c|}{ Setembro/1995 } & \multicolumn{2}{|c|}{ Setembro/1997 } & \multicolumn{2}{|c|}{ Seleção paulista } \\
\hline & Absoluto & $z$ & Absoluto & $z$ & Absoluto & $z$ \\
\hline Amostra & \multicolumn{2}{|l|}{5} & \multicolumn{2}{|l|}{5} & \multicolumn{2}{|c|}{35} \\
\hline Idade (anos) & \multicolumn{2}{|c|}{$14,2 \pm 1,3$} & \multicolumn{2}{|c|}{$16,2 \pm 1,53$} & \multicolumn{2}{|c|}{$20,5 \pm 1,9$} \\
\hline Peso (kg) & $55,4 \pm 9,9$ & 1,37 & $60,1 \pm 10,8$ & 1,53 & $58,5 \pm 6,6$ & 0,96 \\
\hline Altura $(\mathrm{cm})$ & $163,4 \pm 9,5$ & 1,39 & $168,0 \pm 9,8^{1}$ & 1,78 & $162,7 \pm 6,3$ & 0,76 \\
\hline
\end{tabular}

1 Jovens atletas (setembro de $1995 \times 1997)$ : teste $t$ de Student $(p<0,05)$ no momento inicial (set/1995). Também foi utilizado o coeficiente de correlação de Spearman.

Os valores da seleção paulista de handebol adulta feminina $^{21}$ foram utilizados como parâmetros gold standard da aptidão física e indiretamente determinaram o sucesso esportivo. Para enriquecer a discussão também foram empregados os valores de $z$ da seleção brasileira de handebol $^{22}$. Com exceção dos valores de $z$, todos os demais tratamentos foram calculados pelo software Graph Pad Instat (versão 2.0).

\section{RESULTADOS}

Através do coeficiente de variação foi verificado que a distribuição dos valores absolutos (média \pm desvio padrão) diferiu muito pouco entre os dois períodos distintos das avaliações, de $4,6 \%$ a $18,0 \%$. Isto indica que os resultados foram relativamente constantes durante todo o período do estudo. Ao contrário, os grandes desvios padrões em comparação com as médias dos valores relativos (z) não foram normalmente distribuídos, demonstrando variação percentual de $19,4 \%$ a $90,0 \%$, sugerindo o emprego do teste não paramétrico de Wilcoxon para analisar a evolução dos resultados nos dois períodos e o coeficiente de correlação de Spearman, que determinou o nível de associação entre os resultados como os mais apropriados.

Os valores absolutos e relativos das variáveis peso corporal e altura referentes aos dois momentos do estudo e a seleção paulista são apresentados na tabela 1 .

Com exceção do valor absoluto da variável altura, que se diferenciou significativamente $(p<0,05)$ entre os períodos distintos das avaliações, não foram constatadas diferenças estatisticamente significativas entre os valores absolutos das demais associações entre os períodos e a seleção paulista. A mesma característica também foi observada para os valores de $z$.

As jovens atletas apresentaram tendência de resultados absolutos superiores aos da seleção paulista na variável altura (set/1995: $163,4 \mathrm{~cm}$ e set/1997: $168,0 \mathrm{~cm})$ e no peso corporal (somente) no final do estudo $(60,1 \mathrm{~kg})$. Embora não significativos, os valores de $z$ das jovens atletas demonstraram superioridade aos das atletas da seleção paulista, sendo que no final do estudo (set/1997) a diferença foi maior, o que pode sugerir um processo de amadurecimento mais tardio.

Fazendo uma comparação entre os dados das jovens atletas com os apresentados pela seleção brasileira de handebol adulta feminina 
analisada por Gonçalves et al. ${ }^{22}$, com média de idade de $24,0 \pm 3,8$ anos, foi observada similaridade entre os valores de $z$ tanto para o peso corporal, que é de 1,72 , como para a altura, 1,52, neste último também para o valor absoluto.

Como pode ser observado na tabela 2 , seja analisada absoluta ou relativamente, em geral ambos os grupos apresentaram resultados similares nas variáveis de força muscular de membros inferiores.

Houve tendência de maiores valores absolutos e relativos para as jovens atletas nos dois períodos (exceto para IVC, set/1995: 38,4 $\pm 6,6 \mathrm{~cm}$ e set/1997: 2,12) quando comparadas com a seleção paulista, principalmente no caso do teste de impulsão horizontal.

Embora ambos os grupos (jovens atletas e seleção paulista) apresentassem valores de $z$ inferiores aos da seleção brasileira $(3,36)$, as jovens atletas tiveram no momento final do estudo (set/1997) resultados absolutos (220,6 \pm $13,8 \mathrm{~cm})$ similares aos da seleção brasileira adulta $(224,2 \pm$ $11,7 \mathrm{~cm})$. Raso e Bergamo ${ }^{23}$ registraram que atletas de handebol feminino na faixa etária de 13 a 15 anos de idade demonstraram melhor performance no teste de impulsão horizontal que garotas envolvidas apenas em aulas de educação física curricular.

As atletas campeãs mundiais de basquete feminino na Austrália em 1994 apresentaram índice $z$ muito superior aos deste estudo e aos das atletas da seleção paulista, 2,8 para IVS, 3,8 para IVC e 3,7 para $\mathrm{IH}^{4}$. O mesmo pode ser observado nas atletas da seleção brasileira de voleibol feminino com experiência em campeonatos mundiais e olímpicos, que tiveram índice $z$ de $3,3,4,1$ e 3,9 para IVS, IVC e IH, respectivamente ${ }^{24}$, demonstrando que tanto as jovens atletas como as atletas da seleção paulista tiveram menores resultados, independente da medida.

Podem ser observados na tabela 3 os resultados absolutos e os valores de $z$ das variáveis agilidade, velocidade e potência anaeróbica das jovens atletas e da seleção paulista. Devido os testes de agilidade estarem em tempo de performance, quanto menor o tempo, melhor o resultado; e assim, lembramos que o índice $z$ é apresentado com sinal negativo.

Como está indicado na tabela 3 , as jovens atletas apresentaram 88

* $\mathrm{p}<0,05$ valores absolutos de potência anaeróbica no início do estudo (set/1995) estatisticamente superiores $(\mathrm{p}<0,05)$ aos da seleção paulista.

Exceto o que foi descrito anteriormente, tanto os valores absolutos como os relativos das jovens atletas para as variáveis agilidade e potência anaeróbica demonstraram tendência de superioridade nos dois momentos do estudo, mas principalmente em set/1997, quando os resultados relativos foram praticamente o dobro daqueles apresentados pela seleção paulista. Quanto à velocidade, praticamente nenhuma diferença foi observada entre os grupos.

Os resultados de velocidade foram similares aos da seleção brasileira de voleibol feminino $(-1,50)$. Entretanto, os valores de $z$ das jovens atletas, seleção paulista, seleção brasileira de voleibol feminino e da seleção brasileira de basquete masculino $(-1,40$ para agilidade e $-1,30$ para velocidade) foram inferiores aos da seleção brasileira de basquete feminino campeã mundial: $-2,80$ para velocidade ${ }^{4}$. No entanto, esta e todas as outras seleções anteriormente citadas apresentam valores de $z$ para a agilidade muito inferiores aos das jovens atletas: $-3,01$. Assim, as jovens atletas apresentaram melhores resultados na agilidade, independente da idade e sexo.

Em relação aos coeficientes de correlação, as variáveis antropométricas apresentaram valores significativos tanto no peso corporal como na altura, que variaram de alto

TABELA 2

Valores absolutos e índice $z$ das variáveis de força muscular de membros inferiores

\begin{tabular}{|c|c|c|c|c|c|c|}
\hline \multirow[b]{3}{*}{ Variáveis } & \multicolumn{6}{|c|}{ Jovens atletas } \\
\hline & \multicolumn{2}{|c|}{ Setembro/1995 } & \multicolumn{2}{|c|}{ Setembro/1997 } & \multicolumn{2}{|c|}{ Seleção paulista } \\
\hline & Absoluto & $z$ & Absoluto & $z$ & Absoluto & $z$ \\
\hline IVS (cm) & $34,2 \pm 5,1$ & 1,96 & $35,4 \pm 2,9$ & 1,94 & $31,4 \pm 4,0$ & 1,30 \\
\hline IVC (cm) & $38,4 \pm 6,6$ & 2,97 & $41,6 \pm 2,3$ & 2,12 & $39,7 \pm 4,6$ & 2,38 \\
\hline $\mathrm{IH}(\mathrm{cm})$ & $210,4 \pm 9,6$ & 2,77 & $220,6 \pm 13,8$ & 3,21 & $200,8 \pm 13,0$ & 1,91 \\
\hline
\end{tabular}

TABELA 3

Valores absolutos e índice $z$ das variáveis agilidade, velocidade e potência anaeróbica

Jovens atletas

\begin{tabular}{lcccccccc} 
& \multicolumn{2}{c}{ Setembro/1995 } & & \multicolumn{2}{c}{ Setembro/1997 } & & \multicolumn{2}{c}{ Seleção paulista } \\
\cline { 2 - 3 } Variáveis & Absoluto & $\boldsymbol{z}$ & & Absoluto & $\boldsymbol{z}$ & & Absoluto & $\boldsymbol{z}$ \\
SR (seg.) & $10,9 \pm 0,8$ & $-1,97$ & & $10,2 \pm 0,6$ & $-3,01$ & & $10,9 \pm 0,9$ & $-1,50$ \\
50m (seg.) & $8,6 \pm 0,6$ & $-1,35$ & & $8,5 \pm 0,8$ & $-1,38$ & & $8,6 \pm 0,4$ & $-1,50$ \\
40seg. (m) & $239,2 \pm 16,91$ & 1,90 & & $238,4 \pm 25,7$ & 3,02 & & $230,5 \pm 13,0$ & 1,47 \\
\hline
\end{tabular}

1 Jovens atletas (setembro de 1995) x seleção paulista: $p<0,05$ 
$(0,70)$ a muito alto $(0,99)$, como pode ser observado na tabela 4.

Isso significa que $96 \%$ dos valores absolutos (Pearson) da altura obtidos no momento final do estudo (set/1997) podem ser explicados pelos valores observados na primeira avaliação (set/1995), e em $78 \%$ para os valores de peso corporal.

Dados da literatura têm demonstrado que as variáveis antropométricas são as que apresentam os maiores valores de estabilidade, independente do período de observação ${ }^{3}$.

\begin{tabular}{|c|c|c|c|c|}
\hline \multicolumn{5}{|c|}{$\begin{array}{l}\text { TABELA } 4 \\
\text { Valores de correlação (Pearson e Spearman) } \\
\text { e de explicação (R) para os dados absolutos } \\
\text { e relativos das variáveis antropométricas }\end{array}$} \\
\hline & \multicolumn{2}{|c|}{ Peso } & \multicolumn{2}{|c|}{ Altura } \\
\hline & Absoluto & $z$ & Absoluto & $z$ \\
\hline$r$ & $0,88^{*}$ & $0,86^{*}$ & $0,98^{*}$ & $0,93 *$ \\
\hline $\mathrm{R}$ & 0,78 & 0,74 & 0,96 & 0,86 \\
\hline rho & 0,70 & $0,99 *$ & $0,99 *$ & $0,99 *$ \\
\hline
\end{tabular}

TABELA 5

Valores de correlação (Pearson e Spearman) e de explicação (R) para os dados absolutos e relativos da variável força muscular de membros inferiores

\begin{tabular}{lcccccccc}
\hline & \multicolumn{2}{c}{ IVS } & & & \multicolumn{2}{c}{ IVC } & & \multicolumn{2}{c}{ IH } \\
\cline { 2 - 3 } & Absoluto & $\boldsymbol{z}$ & & Absoluto & $\boldsymbol{z}$ & & Absoluto & $\boldsymbol{z}$ \\
$\mathrm{r}$ & $-0,19$ & 0,08 & & 0,65 & 0,73 & & 0,46 & 0,38 \\
$\mathrm{R}$ & 0,36 & 0,006 & & 0,42 & 0,53 & & 0,21 & 0,14 \\
rho & $-0,20$ & 0,10 & & 0,70 & $0,90^{*}$ & & 0,10 & 0,60 \\
\hline
\end{tabular}

${ }^{*} p<0,05$

TABELA 6

Valores de correlação (Pearson e Spearman) e de explicação (R) para os dados absolutos e relativos das variáveis agilidade, velocidade e potência anaeróbica

\begin{tabular}{|c|c|c|c|c|c|c|}
\hline & \multicolumn{2}{|c|}{ SR } & \multicolumn{2}{|c|}{ 40seg. } & \multicolumn{2}{|c|}{$50 \mathrm{~m}$} \\
\hline & Absoluto & $z$ & Absoluto & $z$ & Absoluto & $z$ \\
\hline$r$ & $0,97 *$ & $0,96 *$ & $0,95^{*}$ & $0,94 *$ & $0,88^{*}$ & $0,77 *$ \\
\hline $\mathrm{R}$ & 0,94 & 0,92 & 0,90 & 0,88 & 0,77 & 0,59 \\
\hline rho & $0,99 *$ & $0,99 *$ & $0,90 *$ & $0,90 *$ & $0,90^{*}$ & 0,70 \\
\hline
\end{tabular}

Rev Bras Med Esporte - Vol. 6, № 3 - Mai/Jun, 2000
Brito et al. ${ }^{25}$ verificaram valores de 0,84 no peso corporal e 0,91 na altura de meninos de uma região de baixo nível socioeconômico no período de transição da infância para a adolescência $(9,52 \pm 1,72$ anos a 14,7 $\pm 1,87$ anos). Outros estudos como os de Parizkováa ${ }^{26}$, Araújo et al. ${ }^{27}$ e Matsudo et al. ${ }^{28}$ corroboram os presentes achados, porém por intervalos bem maiores.

Nenhuma das variáveis de força de membros inferiores demonstrou valores estatisticamente significativos. A variável impulsão vertical sem auxílio dos membros superiores (IVS) foi a única que não apresentou estabilidade entre os períodos analisados, tendo somente $36 \%$ e $0,6 \%$ (valores absolutos e relativos, respectivamente) dos valores obtidos na segunda avaliação (set/1997) explicados pelos resultados observados na primeira avaliação (set/1995), como pode ser observado na tabela 5 .

Ao contrário, os coeficientes de correlação da variável impulsão vertical com auxílio dos braços (IVC) variaram de moderado (Pearson: 0,65) a alto (Spearman: 0,70), indicando que $42 \%$ dos valores absolutos e $53 \%$ dos valores relativos alcançados em setembro/1997 poderiam ser explicados pelos valores observados na avaliação de 1995 (tabela 5). No teste de impulsão horizontal foi verificado 0,46 para os valores absolutos e 0,38 para os relativos, enquanto para Spearman esses coeficientes foram de 0,10 e 0,60 , respectivamente.

Brito et al. ${ }^{29}$ verificaram valores similares no coeficiente de correlação linear de Pearson $(0,65)$ do IVC em garotos de uma região de baixo nível socioeconômico na transição da infância $(9,52 \pm 1,72$ anos) para a adolescência (14,7 $\pm 1,87$ anos) e superiores aos do presente estudo quando se analisou a impulsão horizontal $(0,73)$. Outros estudos analisaram a força de membros inferiores somente no período da adolescência. Espenschade ${ }^{30}$ acompanhou um grupo de garotos na faixa etária de 13 a 16 anos e observou valores de 0,48 . Beunen et al. ${ }^{31}$ e Ponnet et al. ${ }^{32}$ verificaram correlações altas durante este período da vida, sendo de 0,71 e 0,87 , respectivamente.

Como pode ser observado na tabela 6 , com exceção do teste de 40seg. que apresentou correlação muito alta e significativa tanto como medido por Pearson $(0,94)$ como por Spearman $(0,90)$, o nível de associação entre os dois momentos distintos das avaliações (set/ $1995 \mathrm{x}$ set/1997) foi muito alto e significativo para as variáveis agilidade (SR) e velocidade $(50 \mathrm{~m})$, tanto para os valores absolutos 
como para os relativos (z). Foi verificado que $94 \%$ e $92 \%$ dos valores absolutos e relativos, respectivamente, obtidos para a agilidade em setembro de 1997 podem ser explicados pelos valores observados na avaliação de setembro de 1995.

Os dados de agilidade e velocidade obtidos no presente estudo são superiores aos apresentados por Branta et al..$^{33}$, de 0,70 , Araújo et $a l .{ }^{17}$, de 0,28 e 0,33, respectivamente, Brito et al. ${ }^{19}$, de 0,50 e 0,55 , na ordem, e também quando são corrigidos pelo peso corporal e pela altura de acordo com Andrade et al. ${ }^{15}$, que verificaram correlações de 0,84 e 0,83 e 0,77 e 0,75 , respectivamente, ou ainda quando são analisados conforme a distribuição na curva de Gauss, em que os valores concentrados abaixo da média $(<-1 \mathrm{DP})$ tiveram correlação de 0,37 , na média $(-1 \mathrm{DP} \leq \mathrm{a} \leq+1 \mathrm{DP})$ de 0,23 e acima da média (>1DP) igual a $0,15^{28}$; sempre lembrando que esses autores acompanharam por intervalos de tempo maiores.

\section{DISCUSSÃO}

Estudos prévios têm demonstrado que o peso e a altura corporal $^{3,14,16,34}$, a agilidade e a velocidade ${ }^{31,33}$, assim como a força de membros inferiores ${ }^{30,32}$, tendem a se estabilizar na transição da infância para a adolescência e no período da adolescência. Entretanto, nenhum estudo analisou as características deste fenômeno em atletas. Assim, o presente estudo é o primeiro a observar a estabilidade das variáveis de aptidão física em atletas com o intuito de verificar se a expectativa de sucesso esportivo anteriormente prognosticada ${ }^{35}$ é mantida durante o período da adolescência.

Embora baseado em um intervalo de tempo relativamente pequeno, os nossos resultados forneceram fortes evidências de que a capacidade de performance tende a se estabilizar durante o período da adolescência e então pode se pressupor que os prognósticos feitos no início deste período foram mais seguros.

Foram observadas correlações de Pearson para os valores absolutos e relativos que variaram de $-0,19$ a 0,98 e de $-0,10$ a 0,93 , respectivamente, enquanto os coeficientes calculados por Spearman foram de $-0,20$ a 0,99 , tanto para os valores absolutos como para os relativos. Bloom ${ }^{2}$ define uma variável estável como sendo aquela que exibe correlação maior que 0,50 para duas medidas obtidas num período mínimo de um ano. Contudo, Malina ${ }^{3}$ descreve que uma correlação menor que 0,30 se caracteriza por uma estabilidade baixa e aquela que apresenta valores entre 0,30 e 0,60 seria considerada moderada.

De acordo com o critério adotado por Malina ${ }^{3}$, presumese que todos os valores superiores a 0,60 apresentem característica de estabilidade alta. Neste caso, com exceção das variáveis IVS, que apresentou estabilidade baixa para os valores absolutos e relativos (Pearson: $-0,19$ e 0,08 ; Spearman: $-0,20$ e $-0,10$, respectivamente), e $\mathrm{IH}$, que foi baixa (Spearman: 0,10 para os valores absolutos) e moderada (Pearson: 0,46 e 0,38, respectivamente; e Spearman 0,60 para z), todas as demais variáveis foram altas e significativas. É interessante notar que a variação de todos os valores significativos obtidos no momento final do estudo (set/ 1997) estariam sendo explicados por $59 \%$ a $96 \%$ dos valores observados na primeira avaliação (set/1995).

A hereditariedade, a variabilidade genética e os fatores ambientais podem determinar os padrões de resposta de um indivíduo ao treinamento. Matsudo ${ }^{4}$ cita que um indivíduo que apresenta resposta tardia, mas alta, pode ter sua capacidade subestimada no início do processo de treinamento, enquanto outro que apresenta uma resposta precoce, porém com posterior progressão baixa, pode ter seu potencial de performance superestimado. Neste sentido, os indicadores de performance relacionados com a detecção de talentos são muito mais dependentes da idade biológica (que pode ser medida através do desenvolvimento das características sexuais secundárias, idade de menarca, ou ainda, pela idade óssea) que da idade cronológica ${ }^{36}$.

Foi muito significativo o fato de quatro das atletas participantes do presente estudo terem sido pré-selecionadas para a seleção brasileira de cadetes na temporada de 1997 , pois confirma o alto nível esportivo e respalda os dados apresentados por nós em outro estudo ${ }^{35}$. Assim, os dados permitem supor que o curto espaço de tempo de acompanhamento entre as medidas (dois anos), o período da vida em que os sujeitos se encontravam (adolescência), a condição de todas já terem tido a maturação biológica (medida através da idade de menarca), associados ao fato de se tratarem de atletas que apresentam valores de aptidão física superiores à média da população e similares aos de atletas de alto nível, sejam hipóteses para explicar a estabilidade dos valores entre os dois períodos.

Malina $^{3}$ comenta que existe uma relação inversamente proporcional entre o período de observação e os valores de correlação; assim, quanto menor o período de tempo de exposição entre as medidas, maior é a correlação, o que explicaria em parte os seus achados. Outra variável que influencia os resultados é o posicionamento do indivíduo em relação aos dados da população, ou seja, indivíduos que apresentam resultados nas extremidades da curva normal tendem a manter a mesma posição durante o período da vida, ao contrário daqueles com valores distribuídos mais centralmente $^{28}$. Isto quer dizer que a possibilidade de sucesso esportivo de uma criança que apresenta uma performance extremamente acima da média $(z \geq 2,5)$ aos 10-12 anos de idade é maior do que aquela que apresenta resulta- 
dos similares aos da média da população $(z<-1,5<z<$ $1,5)$, nas variáveis chaves, estando as duas no mesmo nível de amadurecimento sexual.

O coeficiente de correlação de Spearman foi utilizado principalmente pelo fato de os valores do índice $z$ não terem sido distribuídos normalmente, demonstrando um coeficiente de variação com amplitude de $19,4 \%$ a 90,0\%. Entretanto, mesmo quando estes valores foram analisados pelo coeficiente de correlação linear de Pearson, foi verificado que ambos (Pearson e Spearman) variaram muito pouco, o que indica que os valores foram relativamente similares. Adicionalmente, isso nos faz supor que, independente da forma de apresentação dos valores de correlação (Pearson ou Spearman), parece que a capacidade de performance na maioria das variáveis (com exceção da agilidade e da potência anaeróbica) corrigidas pelo índice $z$ não sofreu efeito do ambiente (treinamento); ou que o impacto do mesmo não foi superior ao do avanço cronológico.

Esta hipótese é respaldada pelos dados referentes à altura corporal das jovens atletas que apresentaram incremento significativo $(\mathrm{p}<0,05)$ nos valores absolutos apresentados em setembro de 1997, quando comparados com os do momento inicial do estudo (set/1995). Contudo, quando os valores absolutos foram calculados de acordo com o índice $z$ esta diferença desapareceu, demonstrando que o incremento ocorrido na altura corporal das jovens atletas durante o período da adolescência é conseqüência apenas de fatores relacionados ao genótipo (como, por exemplo, os processos de crescimento e desenvolvimento) e não necessariamente devido aos fatores ambientais (treinamento).

\section{CONCLUSÃO}

Apesar de esportivamente significativo (três temporadas), o intervalo de tempo é relativamente pequeno em termos biológicos, de modo que os presentes achados devem servir de estímulo a análises futuras de períodos mais longos.

A análise das variáveis da aptidão física tanto em valores absolutos como relativos, sendo neste caso utilizado o índice $z$, demonstrou que os valores obtidos no momento final do estudo (setembro/1997) estavam sendo explicados por aqueles valores observados no momento inicial (setembro/1995). A altura corporal, a agilidade, a potência anaeróbica, o peso corporal e a velocidade foram as variáveis que apresentaram correlações muito altas e significativas, enquanto as variáveis de força muscular de membros inferiores (IVS, IVC e IH) demonstraram valores de correlação de fracos $(0,08)$ a moderados $(0,73)$.

Foi observado que os valores de correlação (Pearson e Spearman) não sofreram efeito do ambiente (treinamento) quando foram corrigidos pelo índice $z$. Isto faz supor que provavelmente a influência do genótipo sobre a maioria das variáveis da aptidão física, com exceção da agilidade e da potência anaeróbica, tenha sido maior que a do ambiente.

Este trabalho é o primeiro a analisar a estabilidade das variáveis da aptidão física em atletas e suporta os resultados de outros estudos realizados com adolescentes não atletas, de que ocorre estabilidade em algumas variáveis na transição da infância para a adolescência e durante o período da adolescência. Finalmente, estes dados permitem concluir que as jovens atletas apresentaram estabilidade nas variáveis antropométricas, agilidade, velocidade e potência anaeróbica durante o período da adolescência. Por estas variáveis serem de fundamental importância na prática do handebol, podem ser estimadas com segurança na adolescência, contribuindo para que a expectativa de sucesso esportivo seja predita em idades precoces.

\section{REFERÊNCIAS}

1. Ästrand PO. Crianças e adolescentes: performance, medidas e educação. Rev Bras Ciências Esporte 1993;7:11-4.

2. Blomm BS. Developing talent in young people. Ballantine, New York, 1985.

3. Malina RR. Tracking of physical activity and physical fitness across the lifespan. Res Q Exerc Sport Suppl 1996;67:48-57.

4. Matsudo VKR. Prediction of future athletic excellence. The child and adolescent athlete. In: Bar-Or O, editor. The encyclopedia of sports medicine. International Olympic Committee, 1996:92-109.

5. Hollmann W, Hettinger T. Idade e treinamento competitivo. In: Medicina do esporte, Editora Manole. São Paulo: 555, 1989.

6. Matsudo VKR. Critérios biológicos para diagnóstico, prescrição e prognóstico de aptidão física em escolares de 7 a 18 anos de idade. Tese apresentada ao concurso público de habilitação à livre-docência na Universidade Gama Filho - área de Medicina, Rio de Janeiro, 1992.

7. Wilson JP, Poster DW, editors. Williams textbook of endocrinology, $7^{\text {th }}$ ed., Philadelphia, W.B. Saunders, 1985.

8. Fischbein S. Onset of puberty in MS and DZ twins. Acta Gen Med Gemellot 1977;26:151-8.

9. Malinowski A. Conceptions of norm and normality in biology of manuals medicine. In: Wolanski N, Szemir M, editors. Studies in human ecology, growth and socioeconomic conditions. Polish Academy of Sciences, Warsaw, 1986.

10. Thomis MAI, Beunen GP, Maes HH, Blimkie CJ, Van Leempute M, Claessens AL, Marshal G, Willems E, Vlietinck RF. Strength training: importance of genetic factors. Med Sci Sports Exerc 1998;30:724-31.

11. Simoneau JA, Lortie G, Boulay MR, Margotte M, Thilbault MC, Bouchard C. Inheritance of human skeletal muscle and anaerobic capacity adaptation to high-intensity intermittent training. Int J Sports Med 1986; 7:167-71

12. Prud'homme D, Bouchard C, Leblanc C, Landry F, Fontaine E. Sensitivity of maximal aerobic power to training is genotype dependent. Med Sci Sports Exerc 1984;16:489-93.

13. Bergamo VR. Talento, treinamento e rendimento no basquetebol feminino. Dissertação apresentada como requisito parcial para a obtenção do Título de Mestre em Educação - área de concentração Educação Motora, UNIMEP, Piracicaba, 1996. 
14. Pate RR, Baranowski T, Dowda M, Trost SG. Tracking of physical activity in young children. Med Sci Sports Exerc 1996;28:92-6.

15. Andrade DR, Matsudo VKR, Brito CFd'A, Figueira Jr. AJ, Matsudo SMM. Weight and height influence on tracking on neuromotor variables in children and adolescents. Med Sci Sports Exerc Suppl 1997; 29:S105.

16. Brito CFD, Andrade DR, Rocha JR, Araújo TL, Matsudo VKR. Estabilidade do nível de aptidão física entre crianças e adolescentes. XX Simpósio Internacional de Ciências do Esporte, São Paulo 1996;111.

17. Araújo TL, Matsudo VKR, Matsudo SMM, Andrade DR, Andrade EL. Tracking of physical fitness of girls at childhood and at adolescence in south coast of Brazil. Med Sci Sports Exerc Suppl 1997;29:S106.

18. Bracco MM, Andrade EL, Brito CFd'A, Matsudo VKR. Influence of nutritional status on stability of physical fitness in boys from low socioeconomical region. Med Sci Sports Exerc Suppl 1997;29:S106.

19. Brito CFD, Andrade DR, Araújo TL, Matsudo VKR. Tracking variability of fitness components in boys from low socioeconomic region. Med Sci Sports Exerc Suppl 1998;30:S149.

20. Matsudo VKR, Rivet RE, Pereira MHN. Standard score assessment on physique and performance of Brazilian athletes in a six tried competitive sports model. J Sports Sci 1987;5:49-53.

21. Carvalho MTC. Perfil de aptidão física de atletas de handebol. XVI Simpósio Internacional de Ciências do Esporte, São Caetano do Sul 1988;26.

22. Gonçalves HR, Osiecki R, Tsuneta P e Zamberlan E. Parâmetros antropométricos, metabólicos e motores em handebolistas de alto nível. Rev Bras Ciência Mov 1991;5:55-9.

23. Raso V, Bergamo V. Estudo comparativo de aptidão física entre escolares e atletas do desporto handebol. Relatório Final do Programa Institucional de Bolsas de Iniciação Científica (PIBIC) do Conselho Nacional de Desenvolvimento Científico e Tecnológico (CNPq), Piracicaba, 1995

24. Figueira Jr AJ, Matsudo VKR. Determination of physical performance of Brazilian volleyball players through z index. In: Coudert J, van Pragh E, editors. Pediatric Work Physiology Supplement, Masson, Paris 1992; 164.

25. Brito CFD, Andrade DR, Rocha JR, Araújo TL, Matsudo VKR. Estabilidade da aptidão física em escolares localizados em diferentes posições da curva normal. Anais do $1^{\circ}$ Congresso Brasileiro de Atividade Física e Saúde, Florianópolis 1997;93.
26. Parizková J. Body fat and physical fitness. Martinus Nijhoff, The Hague, 1977.

27. Araújo TL, Matsudo VKR, Matsudo SMM, Andrade DR, Andrade EL. Estabilidade de aptidão física em meninas de Ilhabela. XX Simpósio Internacional de Ciências do Esporte, São Paulo 1996;144.

28. Matsudo VKR, Matsudo SMM, Andrade EL, Araújo TL. Central and peripheral tracking in girls from low socioeconomical region. Med Sci Sports Exerc Suppl 1997;29:S105.

29. Brito CFD, Andrade DR, Rocha JR, Araújo TL, Matsudo VKR. Physical fitness stability from infancy to adolescence in Brazilian boys. Med Sci Sports Exerc Suppl 1997b;29:S106.

30. Espenschade A. Motor performance in adolescence, including the study of relationships with measures of physical growth and maturity. Monographs of the Society for Research in Child Development 1940;5, $\mathrm{n}^{\circ}$ 24.

31. Beunen G, de Beul G, Ostyn M, Renson R, Simons J, Van Gerven D. Die konstanz motorischer leistungen bei 12- bis 17 jãhrigen jungen. In: Willimczik K, Grosser M, editors. Die motorische entwicklung im kindes- und jugendalter. Schorndorf: Verlag Karl Hofmann 1979;27884

32. Ponnet P, Beunen G, Claessens AL, Lefevre J, Maes H. Stability of athletic performance in untrained boys age 12 to 15 years. In: Claessens A, Lefevre J, Vanden Eynde B, editors. World-wide variation in physical fitness. Leuven: Institute of Physical Education, Katholieke Universiteit Leuven 1993;136-40.

33. Branta C, Haubenstricker J, Seefeldt V. Age changes in motor skills during childhood and adolescence. Exerc Sports Sci Rev 1984;12:467520 .

34. Inman JW, Tremblay MS, Deschenes ARC, Bothwell-Myers CM. Fitness variables track during a five years follow-up study in children with contrasting motor abilities. Med Sci Sports Exercise Suppl 1997; 29:S13.

35. Raso V, Matsudo SMM, Matsudo VKR, Bracco MM. Successful expectation of young handball athletes by z-score strategy. In: Welsman J, Armstrong N, Kirby B, editors. Children and Exercise XIX (Volume II). 1997;155-9.

36. Matsudo SMM, Paschoal VCP, Amancio OMS. Atividade física e sua relação com o crescimento e a maturação biológica de crianças. Cad Nutrição 1997;14:1-12. 